\title{
Adiabatic ring opening in tethered naphthalene and anthracene cycloadducts $\uparrow$
}

\author{
Arun Kumar Sundaresan, ${ }^{a}$ Steffen Jockusch, ${ }^{a}$ Yongjun Li, ${ }^{a}$ Jeffrey R. Lancaster, ${ }^{a}$ Steven Banik, ${ }^{a}$ \\ Paul Zimmerman, ${ }^{b, c}$ James M. Blackwell, ${ }^{c}$ Robert Bristol ${ }^{c}$ and Nicholas J. Turro*a
}

\author{
Received 19th April 2010, Accepted 24th May 2010 \\ First published as an Advance Article on the web 5th June 2010 \\ DOI: 10.1039/c0pp00096e
}

\begin{abstract}
The cycloadducts of tethered naphthalene and anthracene derivatives undergo photochemical ring opening to an electronically excited product with adiabatic yields up to $90 \%$.
\end{abstract}

In an adiabatic photoreaction an electronically excited state, ${ }^{*} \mathrm{R}$, is transformed to an electronically excited product, ${ }^{*} \mathrm{P}$. The overall ${ }^{*} \mathrm{R} \rightarrow{ }^{*} \mathrm{P}$ chemical process occurs entirely on the same excited electronic energy surface. ${ }^{1}$ Most photochemical reactions are not adiabatic, that is, the excited state ${ }^{*} \mathrm{R}$ reverts to the ground state potential energy surface of $\mathrm{R}$ during the course of the reaction so that the photoproduct, $\mathrm{P}$, is formed in its ground state. In an adiabatic photoreaction the photoproduct is formed in its excited state $* \mathrm{P}$. The product $* \mathrm{P}$ can then relax to the ground state by radiative and/or non-radiative pathways. Adiabatic photochemical reactions in which substantial bond breaking and bond making occur are relatively rare, presumably because surface crossings of * $\mathrm{R}$ allow facile deactivation to the ground state and because the energy of $* \mathrm{P}$ is often higher than that of $* \mathrm{R}$. However, adiabatic cycloreversion has been reported for anthracene cycloadducts, ${ }^{2,3}$ and anthracene or naphthalene cycloadducts with benzene $e^{4,5}$ and furan. ${ }^{4}$ This report qualitatively and quantitatively examines the adiabatic contribution to ring opening for the cycloadducts of tethered naphthalene-naphthalene (c1-c3), naphthalene-anthracene (c4) and anthracene-anthracene (c5) compounds shown in Fig. 1.

The primary motivation for examining the chemistry of naphthalene cycloadducts was to use them as photosensitizers for acid generation, a critical part of current $193 \mathrm{~nm}$ photolithography technology. ${ }^{6}$ Our research objective was to use a two-step photochemical (double exposure) process, which would improve the resolution of conventional (one step, single exposure) techniques (see Scheme 1) using a naphthalene-based cycloadduct as a sensitizer. In this scheme, ring opening of the cycloadduct is the first step, forming a stable intermediate (naphthalene). ${ }^{7}$ In a second photochemical event, the intermediate yields a naphthalene chromophore in its excited state that undergoes

${ }^{a}$ Department of Chemistry, Columbia University, New York, NY, 10027, USA.E-mail:njt3@columbia.edu

${ }^{b}$ Intel assignee to Sematech, Austin, TX, 78741, USA

'Intel Corporation, Hillsboro, OR, 97124, USA

$\dagger$ Electronic supplementary information (ESI) available: Synthesis procedures, NMR characterization, additional luminescence spectra. See DOI: $10.1039 / \mathrm{c} 0 \mathrm{pp} 00096 \mathrm{e}$ competing recyclization or quenching by an accessible transparent acid generator (Scheme 1). A study with 2-methoxynaphthalene demonstrated the feasibility of the process using the corresponding cycloadduct. ${ }^{7,8}$ Although 2-methoxynaphthalene is one of the few naphthalenes that undergoes $[4+4]$ cycloaddition, low quantum yields of this reaction necessitated the use of tethered naphthalenes which cyclize efficiently. Structures of the selected tethered naphthalenes and their corresponding cycloadducts discussed in this report are shown in Fig. 1. Although the photochemistry of each tethered naphthalene/anthracene shown in Fig. 1 has been reported, the properties of the cycloadducts are not well established. During the course of our investigations, we discovered that the cycloreversion pathways were to some extent adiabatic. This report compares the extent of adiabatic cycloreversion occurring among the cycloadducts shown in Fig. 1.

Fluorescence spectroscopy is a convenient method to study adiabatic photoreactions. Fig. 2 (left) shows the fluorescence spectrum of 1 , where the structured features between 310 and $360 \mathrm{~nm}$ are assigned to naphthalene fluorescence ${ }^{9-11}$ and the broad band between 370 and $520 \mathrm{~nm}$ is assigned to intramolecular excimer fluorescence. Photoexcitation of the cycloadduct c1 generates a nearly identical fluorescence spectrum to the ringopened form 1. This is a strong indication of the adiabatic nature of the ring opening reaction. To compare the extent of the adiabatic process with the diabatic process, the fluorescence experiments shown in Fig. 2 (left) were performed with identical absorbance values at the excitation wavelength to ensure that equal amounts of photons were absorbed. Comparison of the integrated fluorescence intensities (monomer + excimer) of $\mathbf{1}$ and $\mathbf{c 1}$ indicate that the relative adiabatic contribution in the ring-opening process is approximately $80 \%$. Particular care was taken to ensure that the amount of $\mathbf{1}$ formed in situ during the fluorescence measurements of $\mathbf{c 1}$ was negligible and the absence of a significantly absorbing amount of 1 was confirmed by comparing the UV absorption spectra before and after recording the fluorescence.

Fig. 3 shows schematically the general decay pathways for the excited state of any of the cycloadducts studied using c1 as an example. From the excited cycloadduct, ${ }^{*} \mathbf{c 1}$, the primary photoreaction is the ring opening reaction to produce *1. Analysis of the emission resulting from the photoexcitation of the cycloadducts of compounds $\mathbf{1}-\mathbf{5}$ showed an interesting trend. Based on analysis of the emission data, $* \mathbf{1}$ and $* \mathbf{2}$ were formed from $\mathbf{c 1}$

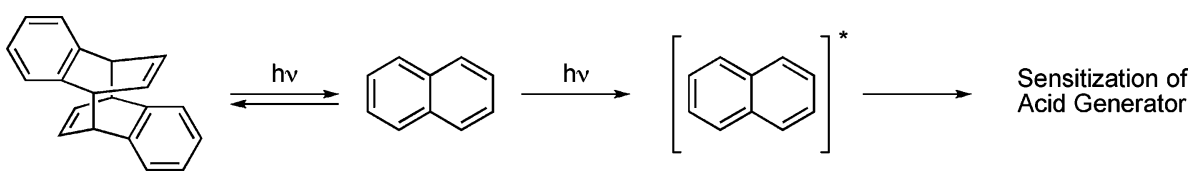

Scheme 1 Schematic representation of the sequential two-photon initiated lithography process. 


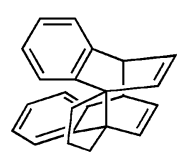

c1<smiles>c1ccc2c(CCCc3cccc4ccccc34)cccc2c1</smiles>

1

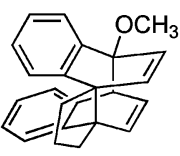

c2<smiles>COc1ccc(CCCc2cccc3ccccc23)c2ccccc12</smiles>

2

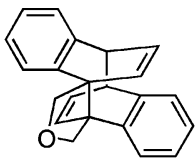

exo-c 3

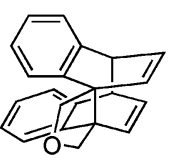

endo-c3<smiles>c1ccc2c(COCc3cccc4ccccc34)cccc2c1</smiles>

3

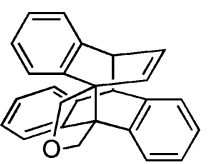

c4

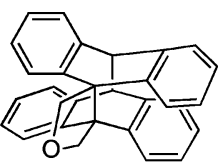

c5<smiles>c1ccc2c(COCc3c4ccccc4cc4ccccc34)cccc2c1</smiles><smiles>c1ccc2c(COCc3c4ccccc4cc4ccccc34)c3ccccc3cc2c1</smiles>

5

Fig. 1 Structures of the cycloadducts (c1 to $\mathbf{c 5}$, top) and the corresponding ring-opened compounds (1 to $\mathbf{5}$, bottom) discussed in this study. (Photocyclization of $\mathbf{3}$ gave two isomers, endo- and exo-, whereas only the endo-isomers were observed for $\mathbf{1}$ and $\mathbf{2}$.)
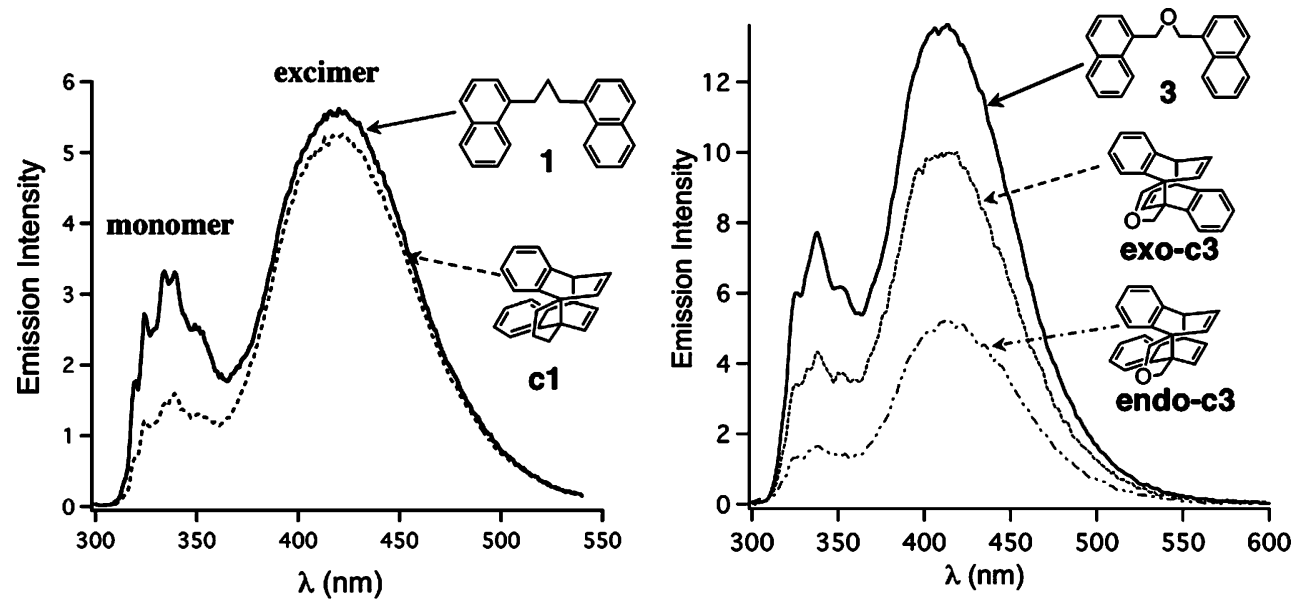

Fig. 2 Left: Emission spectra of 1 (solid line) and c1 (dotted line) recorded in Ar saturated acetonitrile at room temperature. $\lambda_{\text {ex }}=273 \mathrm{~nm}$. The absorbance of $\mathbf{1}$ and $\mathbf{c} \mathbf{1}$ at $\lambda_{\text {ex }}(273 \mathrm{~nm}$ ) was 0.18. Right: Emission spectra of $\mathbf{3}$ (solid line), exo-c3 (dotted line) and endo-c3 (dash-dotted line) recorded in Ar saturated acetonitrile at room temperature. $\lambda_{\mathrm{ex}}=265 \mathrm{~nm}$; absorbance of $\mathbf{3}$, exo-c3 and endo-c3 at $\lambda_{\mathrm{ex}}(265 \mathrm{~nm})$ was 0.20 .

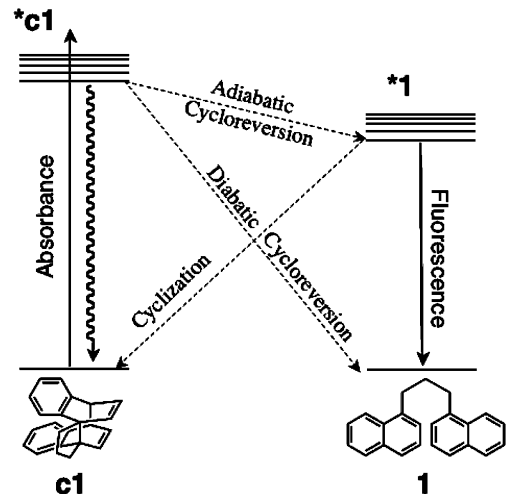

Fig. 3 Schematic representation of the photoreaction of the excited state cycloadduct *c1. Solid lines represent absorption or emission; dashed lines represent reaction.

and $\mathbf{c 2}$ in about $80 \%$ yield, respectively (Table 1). Furthermore, the fraction of adiabatic reaction was larger (90\%) with the anthracene cycloadducts $\mathbf{c 4}$ and $\mathbf{c 5}$ (for emission spectra, see
$\mathrm{ESI} \dagger)$. Although a higher ratio of adiabatic cycloreversion is reported in anthracene adducts (up to $91 \%$ ), ${ }^{15-17}$ similar trends for naphthalene-cycloadducts have not been reported, probably due to poor yield of cycloaddition of naphthalenes. Cycloadducts of naphthalene-furan, ${ }^{4}$ naphthalene-benzene, ${ }^{4,5}$ and naphthalenephthalic anhydride ${ }^{18}$ exhibit adiabatic ring opening, to varying degrees $(0 \%$ for 1-cyanonaphthalene-furan adduct to $63 \%$ for naphthalene-benzene adduct). This study showed that a large fraction of ring opening occurs adiabatically in both naphthalenenaphthalene adducts $\mathbf{c 1}$ and $\mathbf{c 2}$, and naphthalene-anthracene adduct $\mathbf{c 4}$.

Unlike the methylene bridged tethered naphthalenes $\mathbf{1}$ and 2, which photochemically form only the endo-cycloadduct, the ether bridged $\mathbf{3}$ formed two cycloadducts, exo-c3 and endo-c3. ${ }^{12}$ Although the endo-adduct thermally isomerizes to a stable Coperearrangement product, the photochemistry of both compounds is identical. The arrangement of the benzene rings is significantly different in these two isomers; the two rings are almost overlapped in endo-c3 and are farther apart in exo-c3. Contrary to our initial expectations, among all compounds studied, adiabatic 
Table 1 Relative adiabatic contribution during the ring opening of the cycloadducts and fluorescence quantum yields of the corresponding photoproducts

\begin{tabular}{lllllll}
\hline Compound & c1 & c2 & exo-c3 & endo-c3 & c4 & c5 \\
\hline $\begin{array}{l}\text { Relative adiabatic } \\
\text { contribution }\end{array}$ & $80 \%$ & $80 \%$ & $65 \%$ & $32 \%$ & $90 \%$ & $90 \%$ \\
$\Phi_{\text {fluorescence }} /$ open form & & $0.1^{b}$ & $0.27^{b}$ & $0.033^{c}$ & $0.09^{d}$ & $0.03^{e}$
\end{tabular}

${ }^{a}$ Sum of monomer and excimer fluorescence for 1, 2 and 3; compounds $\mathbf{4}$ and $\mathbf{5}$ do not show excimer fluorescence in the conditions used for this experiment. ${ }^{b}$ Determined using 1-methylnaphthalene as standard in argon saturated acetonitrile at room temperature. ${ }^{c}$ Ref. $12 .{ }^{d}$ Ref. $13 .{ }^{e}$ Ref. 14.

ring opening was least efficient for endo-c3 (32\%), while the fraction for exo-c3 was $65 \%$ (Fig. 2, right), marginally lower than c1 and c2. Since the cycloreversion can occur with minimum distortion of the overall structure in any one of the six adducts discussed here, a major deviation from the trend of highly adiabatic ring opening observed with endo-c 3 and to certain extent with exo-c3 is intriguing. Further analysis of similar cycloadducts is being pursued to better understand the role of structure and substitutions in adiabatic ring opening processes.

In conclusion, as demonstrated by quantitative emission spectroscopy, and in contrast to most photochemical reactions, cycloadducts in Fig. 1 undergo photoinduced [4 + 4] retrocycloaddition in an adiabatic manner, that is, the electronically excited state of the cycloadduct, ${ }^{*} \mathrm{R}$, undergoes a ring opening to an electronically excited product, ${ }^{*} \mathrm{P}$, with adiabatic yields of up to $90 \%$. Two general features of the adiabaticity of these reactions for the systems studied are (1) the more or less concerted nature of the reaction which avoids surface crossings and conical intersections along the reaction path to $* \mathrm{P}$ and (2) the exothermicity of the $* \mathrm{R} \rightarrow{ }^{*} \mathrm{P}$ process, which avoids a significant barrier to ${ }^{*} \mathrm{P}$ along the reaction pathway.

\section{Acknowledgements}

The authors thank SEMATECH and the National Science Foundation (Grant CHE 07-17518) for their generous support of this research, and Edward Jackson and Shaun Garratt at SAFC Hitech $\AA$ for providing compounds $\mathbf{4}, \mathbf{5}, \mathbf{c 4}$ and $\mathbf{c 5}$.

\section{References}

1 N. J. Turro, J. McVey, V. Ramamurthy and P. Lechtken, Adiabatic Photoreactions of Organic Molecules, Angew. Chem., Int. Ed. Engl., 1979, 18, 572-586.
$2 \mathrm{~J}$. Ferguson and M. Puza, Electronically excited products in the photodissociation of dianthracene and related compounds, Chem. Phys. Lett., 1978, 53, 215-218.

3 S.-A. Yamamoto, K.-H. Grellmann and A. Weller, Mechanism of the photodissociation of the 9-methylanthracene photodimer, Chem. Phys. Lett., 1980, 70, 241-245.

4 T. Noh, D. Kim and S. Jang, Photoreversion of naphthalene-benzene cyclodimers and naphthalenes-furan cyclodimers, Bull. Korean Chem. Soc., 1997, 18, 357-360.

5 H. Okamoto, M. Kimura, K. Satake and S. Morosawa, Synthesis and Adiabatic Photochemistry of a 1,4-Difluorobenzene - Naphthalene Biplanemer, Bull. Chem. Soc. Jpn., 1993, 66, 2436-2439.

6 S. Lee, K. Jen, C. G. Willson, J. Byers, P. Zimmerman and N. J. Turro, Materials modeling and development for use in double-exposure lithography applications, J. Micro/Nanolith. MEMS MOEMS, 2009, $\mathbf{8}, 011011$.

7 N. A. O'Connor, A. J. Berro, J. R. Lancaster, X. Gu, S. Jockusch, T. Nagai, T. Ogata, S. Lee, P. Zimmerman, C. G. Willson and N. J. Turro, Toward the Design of a Sequential Two Photon Photoacid Generator for Double Exposure Photolithography, Chem. Mater., 2008, 20, 7374 7376.

8 J. S. Bradshaw and G. S. Hammond, Mechanisms of Photochemical Reactions in Solution. XIX. Photodimerization of Methyl $\beta$-Naphthyl Ether, J. Am. Chem. Soc., 1963, 85, 3953-3955.

9 E. A. Chandross and C. J. Dempster, Reversible intramolecular photodimerization of 1,3-bis( $\alpha$-naphthyl)propane, J. Am. Chem. Soc., 1970, 92, 703-704.

10 J. J. McCullough, Photoadditions of aromatic compounds, Chem. Rev., 1987, 87, 811-860.

11 E. A. Chandross and C. J. Dempster, Excimer fluorescence and dimer phosphorescence from a naphthalene sandwich pair, J. Am. Chem. Soc., 1970, 92, 704-706.

12 B. Todesco, J. Gelan, H. Martens, J. Put, N. Boens and F. C. De Schryver, Photochemistry of non conjugated bichromophoric systems photocyclomerization of di-( $\alpha$-naphthylmethyl)ether, Tetrahedron Lett., 1978, 19, 2815-2818.

13 J.-P. Desvergne, N. Bitit, A. Castellan and H. Bouas-Laurent, Study of non-conjugated bichromophoric systems. Part 3. The photocyclomerization of 9-(1-naphthylmethoxymethyl)anthracene and 9-(2furylmethoxymethyl)anthracene. Interest of the $\mathrm{CH}_{2}-\mathrm{O}-\mathrm{CH}_{2}$ link, $J$. Chem. Soc., Perkin Trans. 2, 1983, 109-114.

14 A. Castellan, J.-M. Lacoste and H. Bouas-Laurent, Study of nonconjugated bichromophoric systems, the so-called jaw photochromic materials. Part 1. Photocyclomerization and fluorescence of bis-9anthrylmethyl ethers, J. Chem. Soc., Perkin Trans. 2, 1979, 411419.

15 A. Albini and E. Fasani, Adiabatic and Charge-Transfer Pathways in the Photocleavage of Dimers and Cycloadducts of Some AromaticMolecules, J. Am. Chem. Soc., 1988, 110, 7760-7763.

16 T. Noh, K. Jeon, Y. Jeong, S. Jang and K. S. Min, Synthesis of 9-cyanophenanthrene-furan and -benzene cyclodimers and their reversion, J. Chem. Soc., Perkin Trans. 2, 1999, 1299-1303.

17 N. C. Yang, M. J. Chen and P. Chen, Chemistry of Benzene Anthracene Cyclodimers, J. Am. Chem. Soc., 1984, 106, 7310-7315.

18 M. Kimura, H. Kura, K. Nukada, H. Okamoto, K. Satake and S. Morosawa, Synthesis and photochemistry of 3,6-difluoro-10,11benzopentacyclo [6,4,0.0,0.0]dodeca-4,10-diene, J. Chem. Soc., Perkin Trans. 1, 1988, 3307-3310. 